\title{
Unsteady Roll Moment Control Using Active Flow Control on a Delta Wing
}

\author{
Xiaowei He, Mathieu Le Provost, Xuanhong An and David R. Williams
}

\begin{abstract}
A feedforward controller is designed to attenuate the roll moment coefficients produced by forced roll motion of a delta-wing type model. Active flow control effectors in the form of variable strength pneumatic slot-jets are located along the trailing edge of the model. The control effectors produce a roll moment coefficient proportional to the momentum coefficient. Direct measurements of the roll moment are made with the model in a wind tunnel. Black-box models for the plant and disturbance are identified, and used in the design of the feedforward controller. The effectiveness of the feedforward controller in attenuating disturbance roll moments produced by forced roll maneuvers is evaluated with periodic and pseudo-random maneuvers. Near the design point of the for the controller the root-mean-square value of the net roll moment is four times smaller than the roll moment without control.
\end{abstract}

Keywords Active flow control $\cdot$ Unsteady aerodynamics $\cdot$ Roll control

\section{Introduction}

During landing aircraft fly at low speeds (approximately $30 \%$ above the stall speed) and at high angles of attack (around $\alpha=10^{\circ}-15^{\circ}$ ), conditions that make them susceptible to the adverse effects of turbulence, such as, partial wing stall. Landing on aircraft carriers can be particularly challenging due to the region of strong velocity gradients and turbulence at the approach end of the flight deck, which is known as the 'burble' [3]. To maintain roll control in this environment, conventional aircraft

X. He $\cdot$ X. An $\cdot$ D. R. Williams $(\bowtie)$

Illinois Institute of Technology, Chicago, IL 60616, USA

e-mail: david.williams@iit.edu

M. L. Provost

University California Los Angeles, Los Angeles, CA 90095, USA 
use mechanical ailerons (or elevons) that deflect in opposite directions, producing roll moments by differential lift on the left and right wings. However, elevons lose effectiveness when the flow separates, because the differential lift is reduced.

Control effectors based on active flow control (AFC) techniques offer the potential for increased control authority when the flow is separated. Examples of AFC for vehicle flight control include axisymmetric bluff body trajectory control using synthetic jet actuators, which is being investigated by Lambert et al. [11]. Unsteady lift and pitching moment control on nominally 2-D wings has been explored by Woo et al. [17, 18], An et al. [1], and Reißner [13]. AFC for unsteady lift control in a surging flow wind tunnel was demonstrated by Kerstens et al. [9] on a 3-D semi-circular planform wing. Tailless aircraft control with variable strength pneumatic jets is being investigate by Williams and Seidel [16].

The focus of this paper is the application of AFC to dynamic roll moment control. In particular, we attempt to design a feedforward control algorithm that will attenuate roll moment disturbances created by a forced roll motion of a tailless aircraft model. It is understood that the forced roll motion does not produce unsteady aerodynamics that are equivalent to an aircraft flying through a velocity gradient. However, it is our contention that a controller that effectively attenuates the forced roll moments would also be effective for naturally occurring external flow disturbances. The control approach is based on the assumption that superposition of a plant model response to actuation and a disturbance model will accurately predict the behavior of the aircraft. Identifying low-dimensional models that account for unsteady aerodynamic effects of the external disturbances is explored using the Sparse Identification of Nonlinear Dynamics (SINDy) algorithm introduced by Brunton et al. [2]. This approach allows the important inputs to the disturbance model to be identified. The feedforward controller is constructed from the product of an inverted nominal plant model, a filter, and a disturbance model. The effectiveness of the control approach is evaluated by using periodic and pseudo-random forced roll motion inputs. The measured roll moment coefficients are compared to predicted values.

The hardware used in the experiment is described in Sect.2. The architecture and components of the feedforward controller are presented in Sect.3. The model identification procedures used, including discussion of the SINDy approach are given in Sect. 4. The system performance with and without active flow control is presented in Sect. 5 for periodic and pseudo-random forced rolling motions. The conclusions are discussed in Sect. 6. 


\section{Experimental Setup}

\subsection{Model and Wind Tunnel}

The model used for these measurements is the tailless UAS shown in Fig. 1. The design is a hybrid of the Lockheed-Martin ICE-101 planform [5] with profiles from the NATO SACCON design [8]. The centerline (root) chord of the model is $c_{r}=$ $330 \mathrm{~mm}$ with a span $b=287 \mathrm{~mm}$. The leading edge sweep angle is $\Lambda=65^{\circ}$. The mean aerodynamic chord is $c_{m a c}=214 \mathrm{~mm}$ with a corresponding Reynolds number $R e_{c_{\text {mac }}}=9.6 \times 10^{4}$. The overall mass of the model including sensors is $0.13 \mathrm{~kg}$, and the corresponding moment of inertia about the x-axis of the model is $I_{x x}=$ $3.12 \times 10^{-4} \mathrm{~kg} \mathrm{~m}^{2}$.

Measurements are done in the Fejer Unsteady Flow Wind Tunnel, whose test section length is $2.1 \mathrm{~m}$ and square cross section has dimensions $0.61 \mathrm{~m} \times 0.61 \mathrm{~m}$. The freestream speed was $6.5 \mathrm{~m} / \mathrm{s}$ for all measurements reported. A dSPACE microLabBox system is used to acquire data and provide control at a rate of 1000 samples/sec.

The suction surface of the model is instrumented with 12 surface pressure sensors (AllSensor 1-inch $\mathrm{H}^{2} \mathrm{O}$ ) mounted internally in the upper surface skin of the model. The sensors have a response time of $0.3 \mathrm{~ms}$. Because the distance between the pressure port opening and the sensor is less than $2 \mathrm{~mm}$, the nominal bandwidth of the sensors is on the order of $1 \mathrm{kHz}$, which is more than sufficient to detect the experimental signals of interest that are below $10 \mathrm{~Hz}$.

The roll motion of the model is controlled by a Hitec servo motor (HSB-9360TH). The servo motor is connected to the internal force transducer with a tube that carries instrumentation and active flow control lines. The roll motion amplitude is $\phi= \pm 20^{\circ}$, and the maximum achievable roll frequency is $2.8 \mathrm{~Hz}$. The roll rates used in this study are $f=0.5,1.0,1.5$, and $2.0 \mathrm{~Hz}$, which correspond to dimensionless frequencies $k=\frac{\pi f c}{U}=0.052,0.103,0.155$, and 0.207 . The angle of attack of the model is controlled with a pair of Copley servo tubes. The angles of attack are manually fixed at $\alpha=0^{\circ}, 5^{\circ}, 10^{\circ}, 15^{\circ}$, and $20^{\circ}$ for the current investigation.

The time-varying forces and moments are measured with an ATI Nano 25 force transducer. The transducer is located internally in the model at $0.54 c_{r}$ from the nose of the model. The roll-moment is defined with the positive $\mathrm{x}$-axis aligned with the model centerline and pointing toward the nose. A positive roll corresponds to the right wing moving downward.

\subsection{AFC Actuators as Control Effectors}

The active flow control actuators are a pair of downward blowing slot jets located at the trailing edge of the model as shown in Fig. 2. The flow rates to the left and right side actuators is controlled by the voltage to a pair of Clippard proportional valves (EV-P-10-4050). The slot jet exits are have a width of $0.2 \mathrm{~mm}$ and a length of 


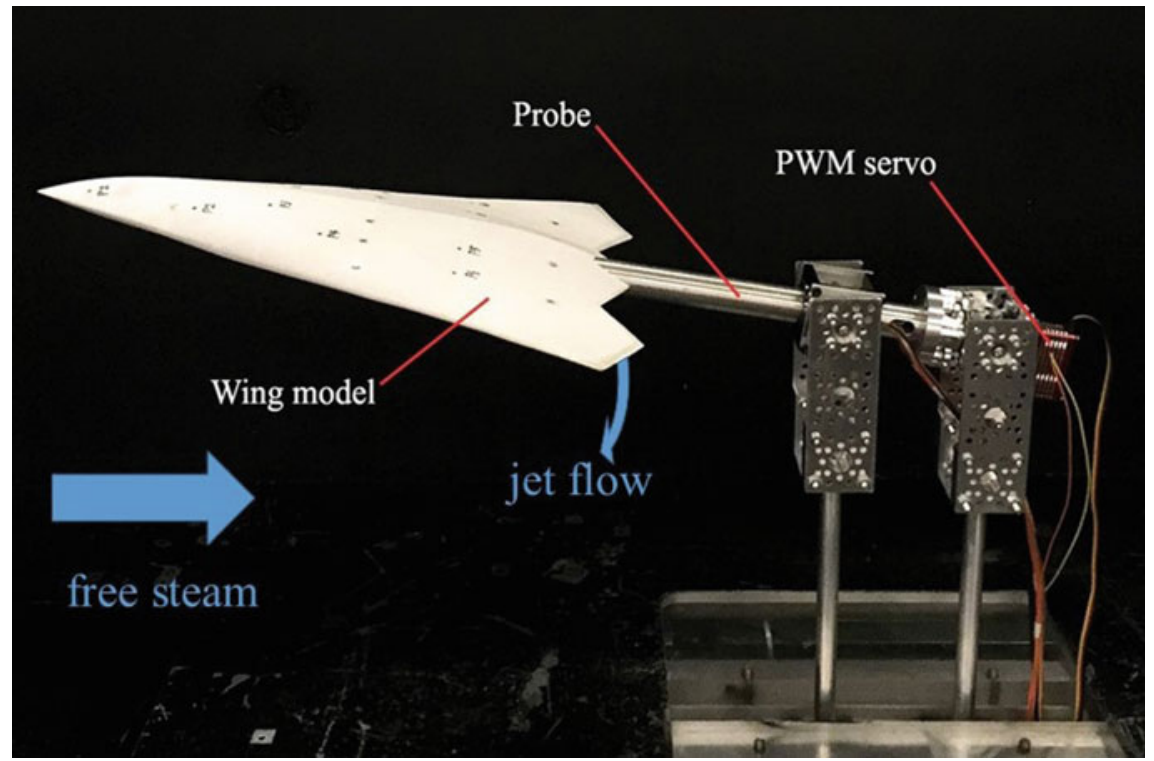

Fig. 1 ICE/SACCON model connected to the roll and pitch mechanism in wind tunnel test section
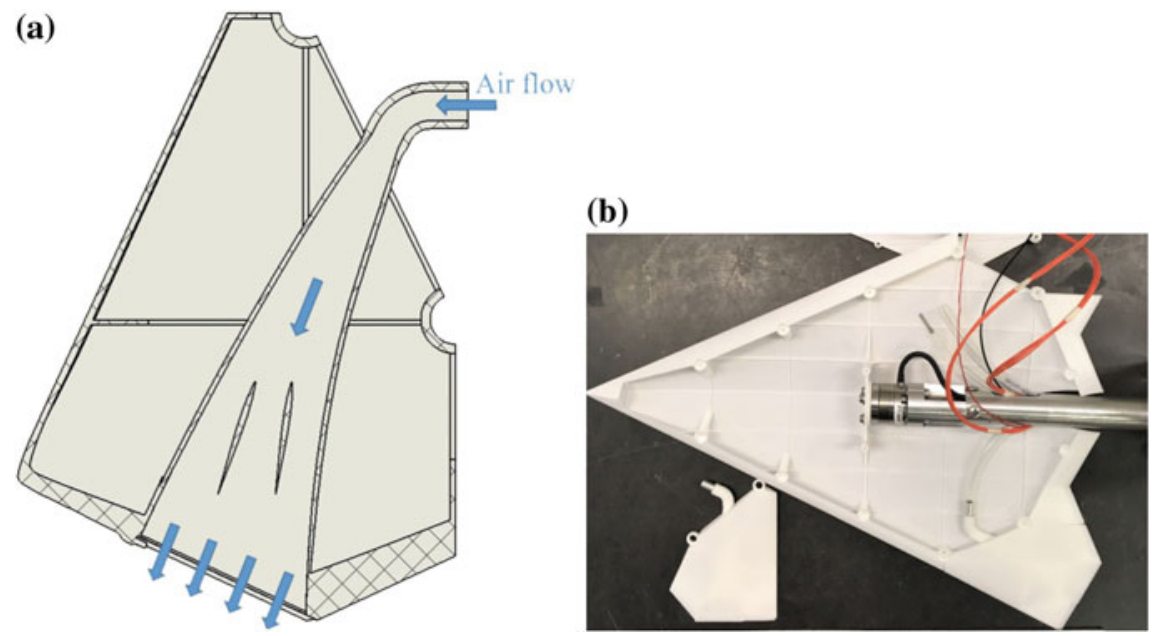

Fig. 2 Active flow control system. a Slot-jet actuator module, b Interior of model showing air supply tubing to actuator 


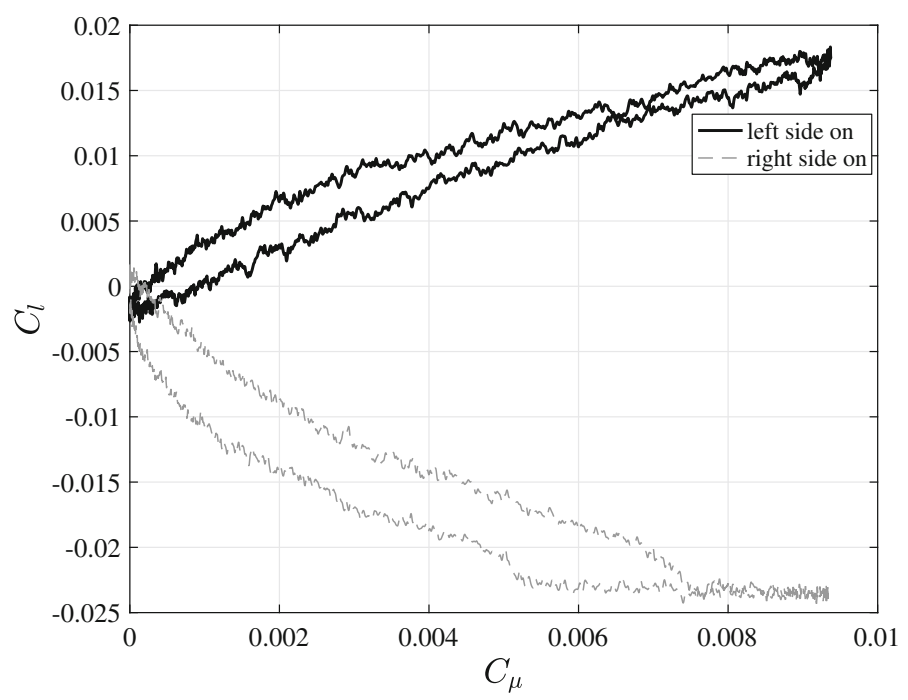

Fig. 3 Steady state calibration of left and right active flow control effectors at $\alpha=20^{\circ}$

$30 \mathrm{~mm}$. The jet exits over the top of a cylindrical surface with a $2 \mathrm{~mm}$ diameter, which produces a downward directed jet by the Coanda effect. The actuators are modular plug-in designs that allow different designs to be used. The strength of actuation is measured by the momentum coefficient, which is defined as $C_{\mu}=\frac{\varrho V_{j e t}^{2} A_{j e t}}{q_{\infty} S}$. Here $q_{\infty}$ is the dynamic pressure, $\mathrm{S}$ is the planform area, $V_{j e t}$ is the jet exit velocity, and $A_{j e t}$ is the slot exit area. The maximum achievable momentum coefficient is $C_{\mu}=0.009$.

Figure 3 shows the roll moment coefficient produced by the left and right wing active flow control actuators. Under steady state conditions the actuators have enough control authority to produce roll moments above $C_{l}=0.015$, which is sufficient for dimensionless roll rates up to $k=0.02$ and roll amplitudes $\pm 30^{\circ}$. The hysteresis is caused by the Clippard valves. The average between the two branches of the hysteresis loops is used for the controller, which ultimately leads to noise in the feedforward controller.

\section{Feedforward Control}

The feedforward control architecture shown in Fig. 4 assumes that the net roll moment $\left(C_{l-\text { measure }}\right)$ of the wing results from the linear superposition of the roll motion disturbance $\left(\Delta C_{l-d}\right)$ and the actuator influence on the plant $\left(C_{l-a c t}\right)$. Even though this approach ignores potential nonlinear interactions between the plant and the disturbance, the linear assumption has proven to be effective in several different 


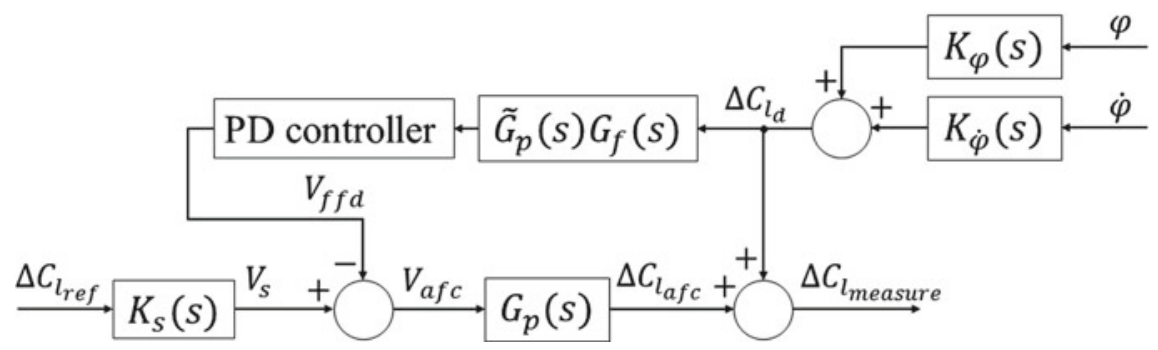

Fig. 4 Block diagram of the feedforward control architecture

active flow control experiments, such as, 2-D bluff body control [7], 3D bluff body gust control [12], 3D wing gust alleviation [9], and stator wake control [10].

The disturbance model $\left(G_{d}\right)$ and plant model $\left(G_{p}\right)$ are identified from experimental measurements. The disturbance model is obtained using an algorithm developed by Brunton et al. [2], which is discussed in Sect.4.1. A third-order black box plant model is identified with the prediction error method. Details are provided in Sect. 4.2. The minimum phase component of the nominal plant model is inverted and combined with a low-pass filter, $G_{f}$ to form the feedforward controller. A PD controller is included with the low-pass filter to act as a lead compensator.

\section{Model Identification}

\subsection{Disturbance Model Identification for the Rolling Wing}

The choice of kinematic inputs to use for the disturbance model is not obvious. The roll angle, roll rate, roll rate derivative, and possible nonlinear combinations of those inputs were all considered to be potentially important in determining the output disturbance roll moment. One way to deal with this uncertainty is to use the Sparse Identification of Nonlinear Dynamics (SINDy) algorithm developed by Brunton et al. [2]. This approach is to assume a large set of candidates exists, and then identify terms that are not active, which are then removed from the model. The procedure is applied at each angle of attack of interest to obtain models for each $\alpha$.

The disturbance model is initially assumed to have the form

$$
\frac{d C_{l}}{d t}=A C_{l}+B f(\phi, \dot{\phi}, \ddot{\phi})
$$

or

$$
\frac{d C_{l}}{d t}=\left[\begin{array}{ll}
A & B
\end{array}\right]\left[\begin{array}{l}
C_{l} \\
f
\end{array}\right]
$$


where $C_{l}$ is the roll moment coefficient, $\phi$ is the roll angle and $f$ is a vector of the input variables. Here, we chose the input $f$ to be

$$
f=[\phi \dot{\phi}, \phi \ddot{\phi}, \ddot{\phi} \ddot{\phi}, \phi, \dot{\phi}, \ddot{\phi}]^{T}
$$

Before seeking the values of $A$ and $B$, Eq. 2 is rewritten in the form of

$$
Y=\Theta(X) \Xi
$$

where $Y$ is the training dataset (experimental dataset in this case), $\Theta(X)$ is a matrix whose elements are a pool of candidate terms, and initally contains all the time series data of $C_{l}, \phi, \dot{\phi}, \ddot{\phi}$, etc. Thus,

$$
\begin{gathered}
Y=\frac{d C_{l}}{d t} \\
\Theta=\left[\begin{array}{c}
C_{l} \text { roll exp } \\
\phi C_{l} \text { roll } \exp \\
\phi \dot{\phi} \\
\phi \ddot{\phi} \\
\ddot{\phi} \ddot{\phi} \\
\phi \\
\dot{\phi} \\
\ddot{\phi}
\end{array}\right]
\end{gathered}
$$

$\Xi$ is the coefficient vector which contains the $A$ and $B$ matrices. The goal is to find a vector $\Xi$ by using sparse regression techniques. One option is to use the least absolute shrinkage and selection operator(LASSO) [15]:

$$
\Xi=\operatorname{argmin}\left\|\Theta \Xi^{\prime}-Y\right\|_{2}+\lambda\left\|\Xi^{\prime}\right\|_{1}
$$

As it is shown in the flow map, Fig. $5, L^{2}$ minimization is performed on Eq. 4 first to generate a initial guess of $\Xi$. Next, if there is any $\Xi_{i}$ smaller than $\lambda$, then we eliminate the corresponding $\Theta_{i}$, update the library of $\Theta$ and repeat the process until all elements inside $\Xi$ satisfy $\Xi_{i}>\lambda$.

The model is identified using a concatenated time series of periodic rolling cases at $0.5,1.0,1.5$ and $2.0 \mathrm{~Hz}$ with $\pm 20^{\circ}$ of amplitude. The sparse coefficient vector computed by the SINDy regression is

$$
\Xi=[-0.9114,0,0,0,0,-0.0208,-0.0028]^{T}
$$

Thus, for $\alpha=10^{\circ}$ the dynamic model is obtained as

$$
\frac{d C_{l}}{d t}=-0.9114 C_{l}-0.0208 \dot{\phi}-0.0028 \ddot{\phi}
$$


Fig. 5 SINDy process flow map

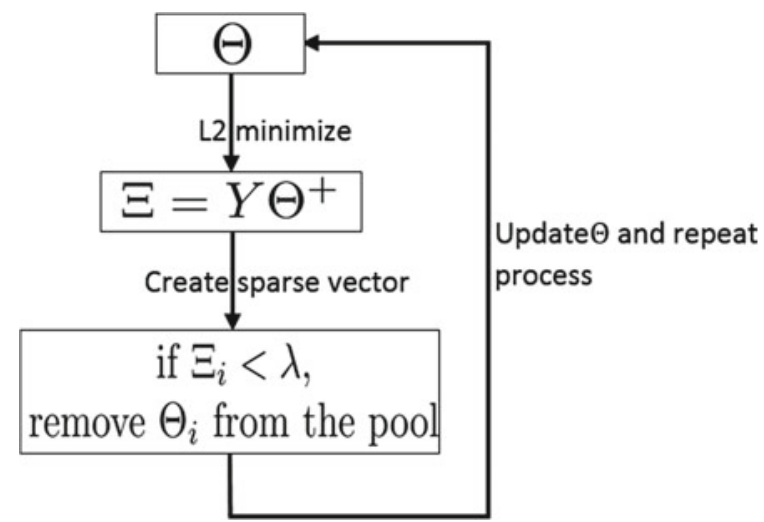

When the angle of attack was increased to $\alpha=20^{\circ}$ then a nonlinear term became significant.

$$
\frac{d C_{l}}{d t}=-6.1038 C_{l}+0.0496 \phi \dot{\phi}+0.1762 \phi-0.976 \dot{\phi}-0.0018 \ddot{\phi}
$$

The model is then validated by a random rolling maneuver. The correlation coefficient between the model estimation and the experimental data is 0.9926 for the $1.5 \mathrm{~Hz}$ periodic cases shown in Fig. 6c, and 0.9253 for the random case shown in Fig. 6d.

\subsection{Plant Model Identification with Actuation}

The plant model predicts the roll moment coefficient response to active flow control actuator input. Conventional system identification techniques that use the prediction error method are used to identify 3rd order models. At each angle of attack a family of 20 different models are identified using pseudo-random binary (prbs) input voltages to the active flow control valves. The binary signal voltage values ranged between 2 and $10 \mathrm{~V}$. The $0-8 \mathrm{~V}$ prbs signal used for input is shown in the lower part of Fig. 7a. The upper part of the figure compares to the measured output roll moment coefficient to the model prediction. The family of plant models is shown in Fig. 7b. There is generally good agreement among the models, except for a few outliers that occurred at low voltage levels. The black dashed line shows the nominal plant model that was obtained by averaging the models not considered to be outliers. The transfer function for the nominal plant at $\alpha=10^{\circ}$ is $\tilde{G}_{p}(s)=\frac{-4.045 \times 10^{-3} s^{2}-0.2959 s+58.35}{s^{3}+56.13 s^{2}+2922 s+44252}$, which has a right-half-plane (RHP) zero at $89 \mathrm{~s}^{-1}$. The RHP zero is analogous to a time delay of approximately $0.022 \mathrm{~s}$, which is short compared to the typical periods of the disturbance motion. 
(a)

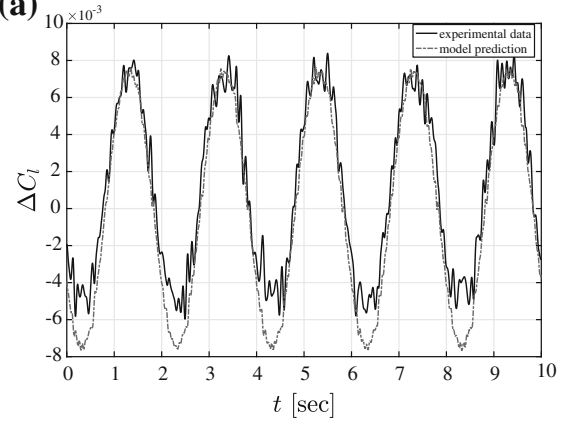

(c)

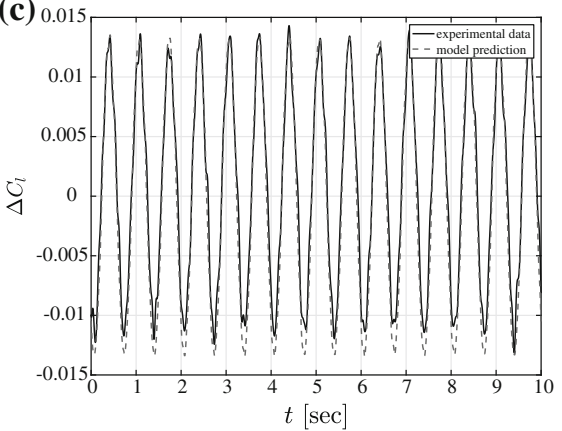

(b)

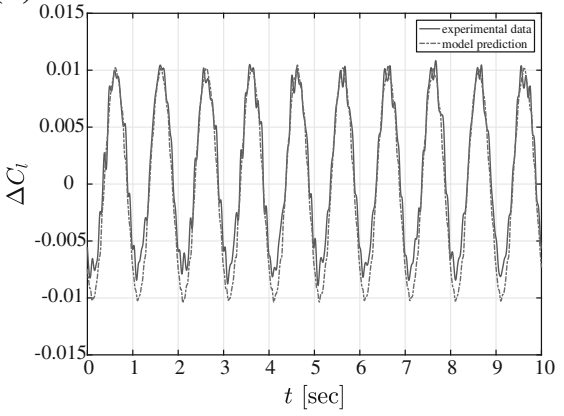

(d)

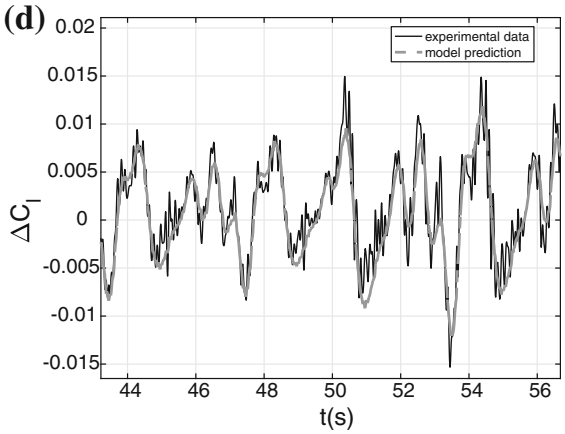

Fig. 6 Comparison of the disturbance model prediction and the experimental data for the periodic rolling at $\alpha=10^{\circ}, \mathbf{a} 0.5 \mathrm{~Hz}, \mathbf{b} 1.0 \mathrm{~Hz}, \mathbf{c} 1.5 \mathrm{~Hz}, \mathbf{d}$ pseudo-random pitching

(a)
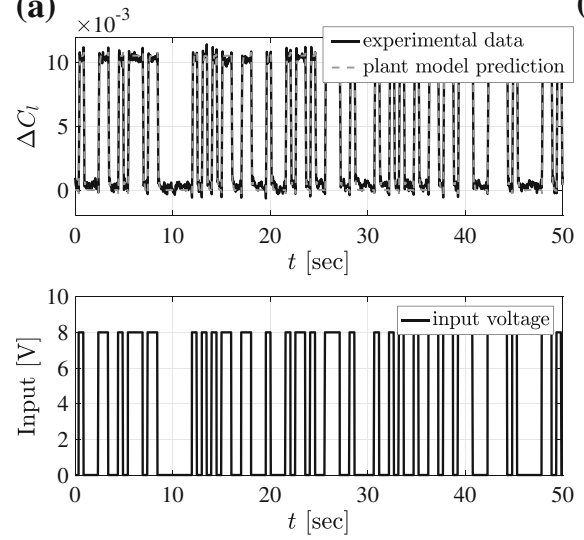

(b)
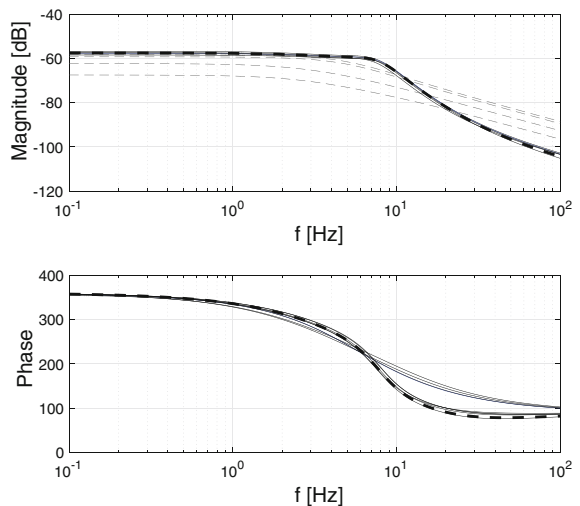

Fig. 7 Plant model identification. a Comparison of measured and modeled prbs signals. b Bode plot of family of plant models and nominal model (heavy dashed) 


\section{Feedforward Control Results}

The minimal phase component of the plant model is inverted and combined with the disturbance model, a low-pass filter, and a PD controller to form the feedforward controller. The objective is to attenuate the effects of periodic and pseudo-random roll motion on the roll moment coefficient. The results are shown in the following two subsections.

\subsection{Roll Moment Attenuation During Periodic Roll Manuevers}

The feedforward control results for forced periodic roll motion at $1 \mathrm{~Hz}(k=0.0102)$ are shown in Fig. 8 at three angles of attack, $\alpha=5^{\circ}, 10^{\circ}$, and $15^{\circ}$. The PD compensator was tuned for the conditions at $\alpha=10^{\circ}$. The uncontrolled roll moment is shown by the dashed line. The solid line shows the roll moment with feedforward control, and the linear model prediction of the roll moment with control is shown by the dash-dot line. At the lowest angle of attack, $\alpha=5^{\circ}$ shown in Fig. 8a, the amplitude of the roll moment oscillations is small (root mean square, r.m.s. $=0.0047$ ). It can be seen that the controller is not very effective in attenuating the roll moment, because the reduced r.m.s. value is only 0.0037 . The simulation predicted the controlled r.m.s. $=0.0016$, which is significantly lower than the value achieved in experiment. The simulation does not account for measurement noise, which is relatively strong at low roll rates. Thus, the inputs to the feedforward controller are low signal-to-noise ratio signals, and it appears that the higher frequency noise is being amplified.

When the angle of attack is increased to $\alpha=10^{\circ}$ as shown in Fig. 8b, the uncontrolled roll moment r.m.s. value increases to 0.0066 . The signal-to-noise ratio is stronger. The controller is more effective at this angle of attack, and the controlled r.m.s. $=0.0016$, which is more than a 4 times reduction in amplitude. The r.m.s. value achieved in simulation is again lower (r.m.s. $=0.0010)$ than experimentally measured.

The case for angle of attack $\alpha=15^{\circ}$ is shown in Fig. $8 \mathrm{c}$. The amplitude of the roll moment coefficient is twice the value seen at $\alpha=10^{\circ}$, and the uncontrolled r.m.s. is 0.0122 . With control the r.m.s. is reduced to 0.0035 , which is 3.5 times smaller than the uncontrolled case.

\subsection{Roll Moment Attenuation During Pseudo-Random Roll Maneuvers}

A sequence of four sine waves with different amplitudes and phases was superposed to create the pseudo-random roll maneuver. Comparisons between the uncontrolled 


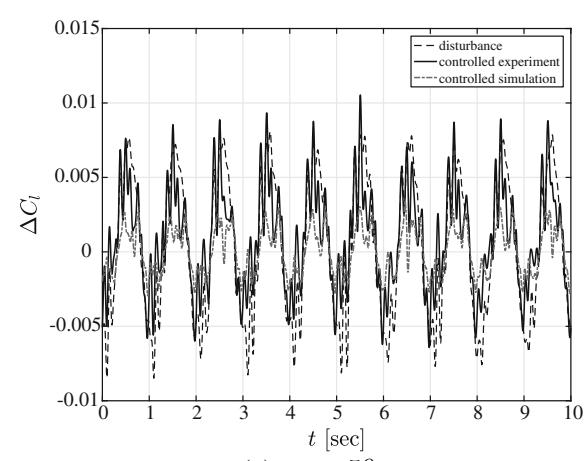

(a) $\alpha=5^{\circ}$

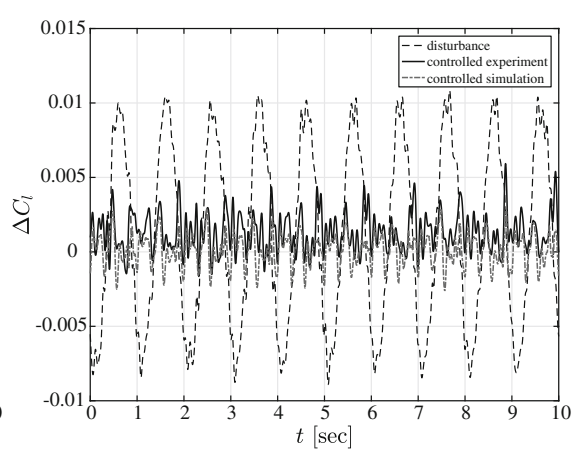

(b) $\alpha=10^{\circ}$

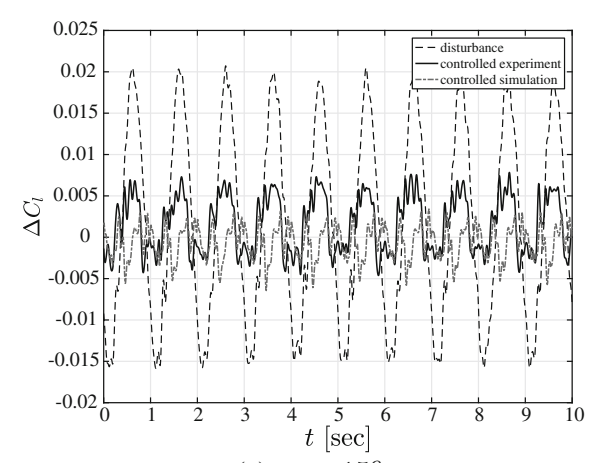

(c) $\alpha=15^{\circ}$

Fig. 8 Periodic roll motion comparison of the measured uncontrolled $C_{l}$ (dashed line), the measured $C_{l}$ with control (solid line), and the predicted response(dash-dot) at $\mathbf{a} \alpha=5^{\circ}, \mathbf{b} \alpha=10^{\circ}, \mathbf{c} \alpha=15^{\circ}$

roll moments, the experimental controlled cases, and the simulation prediction of the controlled cases are shown for three angles of attack in Fig. 9.

The low angle of attack $\alpha=5^{\circ}$ result is shown in Fig.9a. The r.m.s. level of the disturbance roll moment is $\Delta C_{l_{r m s}}=0.0026$. The experimental control has little effect, and only attenuates the disturbance to an r.m.s. level of $\Delta C_{l_{r m s}}=0.0022$. The simulation predicted a significantly lower r.m.s. level of $\Delta C_{l_{r m s}}=0.0012$. From the time series signals it appears that the controller is actually exciting high frequencies in the roll moment.

The data in Fig. $9 \mathrm{~b}$ shows for the $\alpha=10^{\circ}$ angle of attack that the controller becomes more effective. The black-box models used in the controller design were identified at this angle of attack, so it is not surprising that the best level of performance is achieved during the 'on-design' conditions. The uncontrolled r.m.s. level is $\Delta C_{l_{r m s}}=0.0035$. The experimental control case reduces the r.m.s. to $\Delta C_{l_{r m s}}=0.0015$, which is close to the simulated value of $\Delta C_{l_{r m s}}=0.0014$.

In Fig. $9 \mathrm{c}$ the angle of attack is increased to $\alpha=15^{\circ}$. The uncontrolled r.m.s. level more than doubles relative to the $\alpha=10^{\circ}$ case to $\Delta C_{l_{r m s}}=0.0083$. Although the controller is able to reduce the r.m.s. value to $\Delta C_{l_{r m s}}=0.0052$, it is also clear 


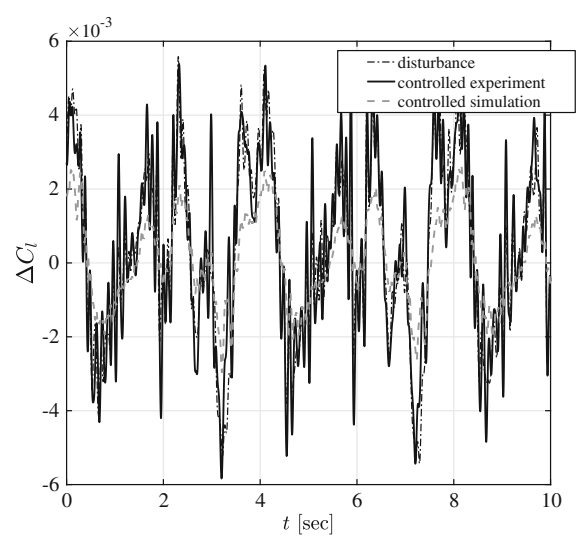

(a) $\alpha=5^{\circ}$

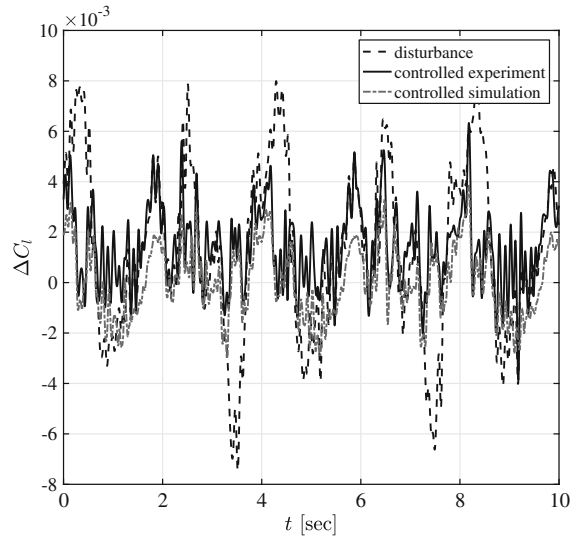

(b) $\alpha=10^{\circ}$

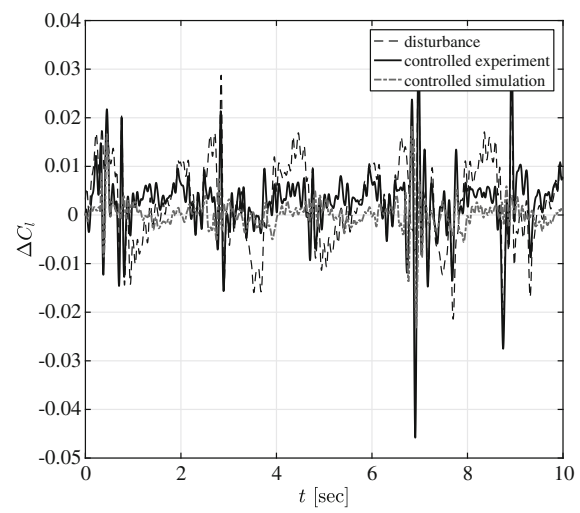

(c) $\alpha=15^{\circ}$

Fig. 9 Pseudo-random motion comparison of the measured uncontrolled $C_{l}$ (dashed line), the measured $C_{l}$ with control (solid line), and the predicted controlled response(dash-dot) at a $\alpha=5^{\circ}$, $\mathbf{b} \alpha=10^{\circ}, \mathbf{c} \alpha=15^{\circ}$

from the time series data that the controller is exciting higher frequencies. Large amplitude spikes in $\Delta C_{l}$ are seen near $t=3,7$, and $9 \mathrm{~s}$, which come from the feedforward controller. In some cases the spike amplitudes exceed the amplitude of the uncontrolled roll moment, so the controller is making things worse.

The next step is to include feedback in the control architecture. So far that has not been attempted, because a suitable surrogate signal for the instantaneous roll moment has not been identified. The current configuration of surface pressure sensors is capable of identifying the disturbance roll moment, but they are not able to identify the roll moment associated with the actuator. 


\section{Conclusion}

Active flow control is used to attenuate the roll moment coefficient produced by forced rolling maneuvers with a $\Delta$-wing (ICE/SACCON) aircraft model. A feedforward controller is designed based on low-dimensional models for the roll moment response to actuation and roll motion. The disturbance model is identified using the SINDy optimization algorithm. The plant model for the response to actuation is identified using the prediction error method. The control is applied during forced periodic and quasi-random oscillatory maneuvers of the model. The controller is able to reduce the r.m.s. level of the roll moment coefficient by a factor of four in the periodic $(1 \mathrm{~Hz}$, $k=0.102$ ) case at $\alpha=10^{\circ}$, which is the 'on-design' condition for the controller. Controller performance is degraded at angles of attack both above and below the design point, but is still effective in reducing the r.m.s. value of the roll moment coefficient for both periodic and pseudo-random forced disturbances. In the case of pseudo-random forcing the controller acted to excite higher frequency disturbances.

Acknowledgements The authors gratefully acknowledge support of the Office of Naval Research (ONR) under Grant N001416-1-2622 with Dr. Ken Iwanski and Dr. Brian Holm-Hansen as program managers.

\section{References}

1. An, X., Grimaud, L., Williams, D.R.: Feedforward control of lift hysteresis during periodic and random pitching maneuvers. In: King, R. (ed.) Active Flow and Combustion Control 2014. NNFM, vol. 127, pp. 55-69. Springer, Heidelberg (2015). https://doi.org/10.1007/978-3-31911967-0_4

2. Brunton, S.L., Proctor, J.L., Kutz, J.N.: Discovering governing equations from data by sparse identification of nonlinear dynamical systems. Proc. Nat. Acad. Sci. 113(15), 3932-3937 (2016). Nat. Acad. Sci

3. Cherry, B.E., Constantino, M.M.: The burble effect: Superstructure and flight deck effects on carrier air wake. In: Proceedings of the American Society of Naval Engineers Launch and Recovery Symposium 2010, Launch, Recovery and Operations of Manned and Unmanned Vehicles from Marine Platforms, Arlington, VA (2010), http://www.dtic.mil/dtic/tr/fulltext/ us/a527798.pdf

4. Cook, M.V.: Flight Dynamics Principles: A Linear Systems Approach to Aircraft Stability and Control, pp. 20-21. Butterworth-Heinemann (2012)

5. Gillard, W.J.: Innovative control effectors (Configuration 101). Technical Report AFRL-VAWP-TR-1998-3043. Air Force Research Laboratory Wright Patterson (1998)

6. Gursul, I.: Review of unsteady vortex flows over slender delta wings. J. Aircr. 42(2), 299-319 (2005). https://doi.org/10.2514/1.5269

7. Henning, L., Pastoor, M., King, R., Noack, B.R., Tadmor, G.: Feedback control applied to the bluff body wake. In: King, R. (ed.) Active Flow Control. NNFM, vol. 95. Springer, pp. 369-390 (2007)

8. Huber, K.C., Vicroy, D., Schütte, A., Hübner, A.: UCAV model design and static experimental investigations to estimate control device effectiveness and stability and control capabilities. In: AIAA Paper 2014-2002 (2014) 
9. Kerstens, W., Pfeiffer, J., Williams, D.R., King, R., Colonius, T.: Closed-loop control of lift for longitudinal gust suppression at low Reynolds numbers. AIAA J 49(8), 1721-1728 (2011). https://doi.org/10.2514/1.J050954

10. Kiesner, M., King, R.: Multivariable closed-loop active flow control of a compressor stator cascade. AIAA J. (2017). https://doi.org/10.2514/1.J055728

11. Lambert, T.J., Vukasinovic, B., Glezer, A.: Aerodynamic flow control of wake dynamics coupled to a moving bluff body. In: AIAA Paper 2016-4081 (2016). https://doi.org/10.2514/6. 2016-4081

12. Pfeiffer, J.: Closed-loop active flow control for road vehicles under unsteady cross-wind conditions. Ph.D. Thesis, Technische Universität Berlin (2015)

13. Reißner, F.: Hysteresis modeling and comparison of controller effectiveness on a pitching airfoil. Master's thesis, Technische Universität Berlin (2015)

14. Taylor, G., Wang, Z., Vardaki, E., Gursul, I.: Lift enhancement over flexible nonslender delta wings. AIAA J. 45(12), 2979-2993 (2007)

15. Tibshirani, R.: Regression shrinkage and selection via the lasso. J. R. Stat. Soc. Ser. B (Method) JSTOR, 267-288 (1996)

16. Williams, D.R., Seidel, J.: Crossed-actuation AFC for lateral-directional control of an ICE101/Saccon UCAV. In: AIAA Paper 2016-3167 (2016)

17. Woo, G., Crittenden, T., Glezer, A.: Transitory control of a pitching airfoil using pulse combustion actuation. In: AIAA Paper 2008-4324 (2008)

18. Woo, G., Crittendon, T., Glezer, A.: Transitory separation control of dynamic stall on a pitching airfoil. In: King, R. (ed.), Active Flow Control II. NNFM, vol. 108, pp. 3-18. Springer, Heidelberg (2010) 